\title{
The Association of Human Leukocyte Antigens Complex with Type 1 Diabetes in the Omani Population
}

Mohammed Al-Balushi, ${ }^{1}$ Samiya Al-Badi, ${ }^{2}$ Saif Al-Yaarubi, ${ }^{3}$ Hamad Al-Riyami, ${ }^{4}$ Azza Al-Shidhani, ${ }^{3}$ Shaima Al-Hinai, Ali Alshirawi, ${ }^{5}$ Sidgi Hasson, ${ }^{1}$ Elias Said, ${ }^{1}$ Ali Al-Jabri, ${ }^{1}{ }^{*}$ Aliya Al Ansari ${ }^{2}$

\begin{abstract}
Objectives: Identification of the high risk alleles, genotypes and haplotypes of the human leukocyte antigens (HLA) in different populations is beneficial for understanding their roles in type 1 diabetes (T1D) pathogenesis and intervention practices. This study aimed to identify T1D-associated HLA gene alleles in the Omani population. Methods: The present case-control study included 73 diabetic seropositive children (mean age $9.08 \pm 3.27$ years) attending the paediatric clinic at Sultan Qaboos University Hospital in Muscat, Oman, and 110 healthy controls. $H L A-A,-B,-C,-D R B 1$ and $-D Q B 1$ genes were genotyped using a sequence-specific primer polymerase chain reaction (SSP-PCR). Results: Two HLA class I alleles $(B * 08, B * 58)$ and three class II alleles (DQB1*02, DRB1*03 and DRB1*04) were associated with T1D susceptibility, while one class I ( $B * 51)$ and three class II ( $D Q B 1 * 05, D Q B 1 * 06$ and $D R B 1 * 16)$ alleles were associated with T1D protection. $H L A-D R B 1^{*} 03$ and $D Q B 1^{*} 02$ alleles showed the strongest risk association among all alleles. Six $D R B 1$ residues $\left(E^{9}, \mathrm{~S}^{11}, \mathrm{~S}^{13}, \mathrm{Y}^{30}, \mathrm{~V}^{70}\right.$ and $\left.\mathrm{K}^{71}\right)$ were significantly associated with T1D susceptibility. Heterozygous genotypes, $H L A-D R B 1 * 03 / * 04$ and $D Q B 1 * 02 / * 03$ were significantly associated with T1D susceptibility $(P<0.0001$, odds ratio $[\mathrm{OR}]=63.21$ and $P=0.02$, OR $=3.63$, respectively). Furthermore, a significant combined action of $D R B 1 * 03-D Q B 1 * 02$ haplotype in T1D risk $(P=0.000176$, OR $=15)$ and $D R B 1 * 16-D Q B 1 * 05$ haplotype in protection $(P=0.0312$, OR $=0.48)$ was detected. Conclusion: Known HLA class II gene alleles are associated with T1D in Omani children.
\end{abstract}

Keywords: Type 1 Diabetes; Human Leukocyte Antigens; Genotype; Alleles; Residues; Haplotypes, Case-Control Study; Oman.

\section{AdVANCES IN KNOWLEDGE \\ Human leukocyte antigens class II alleles (DQB1"02, DRB1"03 and DRB1*04) are the major genetic risk factors for type 1 diabetes (T1D) in Omanis. \\ Combined action in DRB1*16-DQB1*05 haplotype is associated with T1D protection. \\ Combined action in DRB1*03-DQB1*02 haplotype is associated with T1D risk.}

Application to Patient Care

The associated gene alleles can be used for disease prediction and intervention

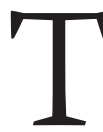
YPE 1 DIABETES (T1D) IS A COMMON, incurable, chronic childhood autoimmune disease, with an estimated incidence increase of $9.5 \%$ globally. ${ }^{1}$ It is a complex disease that develops due to the collective contribution of genetic, epigenetic and environmental factors. ${ }^{2}$ Both cellular and humoral adaptive immune mechanisms are implicated in T1D. The destruction of $\beta$-cells driven by self-reactive CD $8+$ and CD4+ T cells leads to total insulin deficiency. ${ }^{3}$ Autoantibodies to pancreatic islet $\beta$-cell autoantigens are detected prior to disease development and are used as biomarkers for $\beta$-cell dysfunction and T1D progression. ${ }^{4}$ Determining the associated environmental triggers, autoimmune mechanisms and predisposing genetic background hold potential for interventions through prediction, prevention or the slowing down of disease progression.
The estimated heritability of T1D is high ( 0.53 to 0.92 on the liability scale) and familial and populationbased genetic studies have identified more than 60 genes that are responsible for about $80 \%$ of the disease heritability. ${ }^{5}$ Most of the T1D genetic predisposition (60\%) is attributed to the human leukocyte antigen (HLA) class I and class II genes, which are present in the major histocompatibility complex (MHC) region and encode for proteins that present antigenic peptides for CD8+ and CD4+ T cells, respectively. ${ }^{6}$

Markedly $45 \%$ of the genetic predisposition is attributed to HLA class II genes and they are, thus, considered a major genetic risk determinant for T1D.7 The strongest T1D risk is associated with the $D R B 1, D Q A 1$ and $D Q B 1$ gene alleles and there is cumulative supporting evidence for the role of $D R B 1$ and $D Q B 1$ genes in combination as a haplotype. ${ }^{8}$ 
Among European populations, more than $95 \%$ of T1D cases have DR3 (HLA-DRB1*0301-DQB1*0201) or DR4 (HLA-DRB1*04-DQB1*0302) genes.? The same gene alleles and haplotypes associated with HLA susceptibility and protection were reported among Arab populations. ${ }^{9}$

With the current knowledge about autoantigens, genetic risk alleles and biomarkers, disease interventions are more informed and can be considered at three stages: prior to the development of autoimmunity (primary prevention), after autoimmunity is recognised (secondary prevention) or after diagnosis, if significant number of $\beta$-cells are available (tertiary prevention). ${ }^{4}$

In a study conducted over two years on Omani children with T1D (age range: 9 months -14 years), yearly incidence rates were found to be 2.45 and 2.62 per 100,000 children in 1993 and 1994, respectively. ${ }^{10}$ The reported gender-specific yearly incidence rates among boys and girls were 3.23 and 1.99 per 100,000 in 1993 and 2.91 and 1.95 per 100,000 in 1994, respectively. During the two years when this study was conducted, higher age-specific incidence rates were observed in children belonging to the 10-14 years age group compared to younger age groups. Furthermore, a retrospective analysis (June 2006 to May 2013) of 144. T1D Omani children reported a highly prevalent family history of T1D among these patients (22\%). ${ }^{11}$

The incidence of T1D is relatively less among the Omani population when compared to other Arab populations. Furthermore, ketoacidosis was also reported to be less among the Omani population. ${ }^{11}$ Although the Omani population is genetically related to Mediterranean and West-Asian populations, the high frequency of $H L A-D R 2$ and $-D Q 1$ alleles (DRB1*15 and DRB1*16, and DQB1*05 and DQB1*06, respectively) was suggested as a genetic protection factor against T1D in this population. ${ }^{12-14}$ However, it remains to be elucidated whether this is indeed true or whether this observation is attributed to the low frequency of risk alleles.

The following study was conducted with the aim of identifying the potential HLA gene alleles associated with T1D risk and/or protection in Omani patients with T1D through comparison to a set of healthy controls.

\section{Methods}

A total of 100 Omani diabetic patients attending the paediatric clinic at SQUH were included in the study based on their medical records (mean age $9.31 \pm 3.27$ years; $47 \%$ male and $53 \%$ female). None of the patients had another autoimmune disease or syndrome. The diagnosis of T1D was confirmed by the presence of diabetes autoantibodies to islet cell (ICA) and glutamic acid decarboxylase (GADA) and the family history of T1D and T2D was recorded for each case.

Peripheral venous blood samples $(5 \mathrm{~mL})$ were collected in ethylenediaminetetraacetic acidanticoagulated vacutainer tubes and stored at $-20^{\circ} \mathrm{C}$. HLA data of 110 healthy potential bone marrow stem cell donors (mean age $10.77 \pm 3.36$ years; $51 \%$ male and $49 \%$ female) from the national HLA database were used as the healthy population control. DNA was extracted from whole blood samples using QIAamp ${ }^{\circ}$ DNA Medi Kit (Qiagen, Hilden, Germany) according to the manufacturer's instructions. DNA concentration and purity was measured using a NanoDrop ${ }^{\text {max}} 2000$ spectrophotometer (Thermo Fisher Scientific Inc., Waltham, USA). The extracted DNA (20-35 ng/ $\mu \mathrm{L}$ ) was HLA genotyped for $H L A-A,-B,-C$, $-D R B 1$ and $-D Q B 1$ loci using a commercial, sequencespecific primer polymerase chain reaction (SSP-PCR) following the manufacturer's protocol. The generated genotype data were at low resolution.

Agarose gel (1.3\%) electrophoresis was used to detect the amplified PCR product. The gel was visualised using the gel documentation system Ingenious 3 with GeneSys software (Syngene International Ltd., Bangalore, India). The appearance of the internal control bands in all lanes indicated successful amplification of the studied DNA. Negative control wells were checked for contamination. HLA genotypes for each locus were identified using the Olerup SSP score software, Version 5.00.72.5T (CareDX, Brisbane, USA).

Hardy-Weinberg equilibrium tests were conducted for each locus using the basic statistics tool (one-locus summary; HLA-net, University of Geneva, Switzerland) Alleles at each locus were considered in Hardy-Weinberg equilibrium if the observed and expected (estimated) frequencies did not differ significantly $(P>0.05)$. Tests for allele associations, zygosity, independence, difference in association, combined action, interaction and linkage disequilibrium (LD) were conducted using Python for HLA (Python Software Foundation, Wilmington, USA). ${ }^{15}$

The comparison of allele frequencies was performed using Fisher's exact test. The $P$ value for each test was corrected for multiple comparisons by the false discovery rate. Adjusted $P$ values less than 0.05 were considered statistically significant. The strength of the association between HLA antigens and T1D was determined by the odds ratio (OR). An OR $\geq$ 1.5 was associated with susceptibility and a value $\leq 0.5$, with resistance. Additionally, tests for pockets with significant residue association were conducted using 
the SKDM human leukocyte antigen tool (Slashdot Media LLC, San Diego, USA). ${ }^{16}$

This study was approved by the Ethics Research Committee of the College of Medicine and Health Sciences at Sultan Qaboos University, Muscat, Oman. A written informed consent to use the participants' blood sample for research purpose was obtained from the guardians of all the participants enrolled in the study.

\section{Results}

Out of the 100 T1D patients screened initially, 73 (73\%; mean age $9.08 \pm 3.27$ years; $41.1 \%$ male and
$58.9 \%$ female) were included in the study as they were seropositive for GADA and/or ICA autoantibodies. A total of 26 patients (26\%) were seronegative (mean age $9.77 \pm 3.25$ years; $61.5 \%$ male and $38.5 \%$ female), of which three patients (two males and one female) were heterozygous for mutations in different genes (KLF11, WFS1 and HNF1A). Approximately 23\% of the seropositive cases had a family history of T1D, while $59 \%$ had a family history of T2D. About 19\% of the seronegative cases had a family history of T1D, while $54 \%$ had a family history of T2D. One patient was excluded as no antibodies were reported in their test results.

Table 1: Distribution of significantly associated human leukocyte antigen alleles in type 1 diabetes cases $(n=73)$ and controls $(\mathrm{n}=110)$

\begin{tabular}{|c|c|c|c|c|c|c|c|}
\hline Allele & Cases in \% & Control in \% & $P$ value & $\begin{array}{l}\text { Odds } \\
\text { ratio }\end{array}$ & L95 & U95 & $\begin{array}{l}\text { Adjusted } P \\
\text { value }\end{array}$ \\
\hline \multicolumn{8}{|c|}{ Susceptibility } \\
\hline$D R B 1 * 03$ & 49.32 & 16.36 & $2.30 \times 10^{-11}$ & 4.97 & 3.07 & 8.06 & $9.19 \times 10^{-11}$ \\
\hline$D Q B 1 * 02$ & 59.59 & 29.82 & $2.44 \times 10^{-8}$ & 3.47 & 2.24 & 5.39 & $9.76 \times 10^{-8}$ \\
\hline$D R B 1 * 04$ & 19.86 & 8.18 & $1.40 \times 10^{-3}$ & 2.78 & 1.48 & 5.23 & $2.70 \times 10^{-3}$ \\
\hline$B * 08$ & 19.18 & 8.64 & $4.00 \times 10^{-3}$ & 2.51 & 1.34 & 4.69 & $1.82 \times 10^{-2}$ \\
\hline$B * 58$ & 14.38 & 6.36 & $1.72 \times 10^{-2}$ & 2.47 & 1.21 & 5.04 & $2.86 \times 10^{-2}$ \\
\hline \multicolumn{8}{|l|}{ Resistance } \\
\hline$D Q B 1 * 06$ & 0.68 & 11.47 & $3.20 \times 10^{-5}$ & 0.05 & 0.01 & 0.40 & $6.40 \times 10^{-5}$ \\
\hline$D Q B 1 * 05$ & 26.03 & 46.79 & $7.19 \times 10^{-5}$ & 0.40 & 0.25 & 0.63 & $9.59 \times 10^{-5}$ \\
\hline$D R B 1 * 16$ & 20.55 & 32.73 & $1.24 \times 10^{-2}$ & 0.53 & 0.33 & 0.87 & $1.65 \times 10^{-2}$ \\
\hline$B * 51$ & 8.90 & 19.09 & $7.30 \times 10^{-3}$ & 0.41 & 0.21 & 0.80 & $1.82 \times 10^{-2}$ \\
\hline$D R B 1 * 15$ & 3. 42 & 8.64 & $5.38 \times 10^{-2}$ & 0.38 & 0.14 & 1.03 & $5.38 \times 10^{-2}$ \\
\hline
\end{tabular}

Association test performed using the PyHLA programme.

Table 2: Zygosity test results for the associated human leukocyte antigen alleles in 73 diabetic seropositive children versus healthy controls

\begin{tabular}{|c|c|c|c|c|c|c|}
\hline Allele & Hom_P & Hom_OR & Het_P & Het_OR & $\mathrm{Zyg} \_P$ & Zyg_OR \\
\hline$D R B 1 * 03$ & 0.43 & 0.63 & $1.05 \times 10^{-12}$ & 0.07 & $2.27 \times 10^{-5}$ & 8.22 \\
\hline$D Q B 1 * 02$ & 0.32 & 1.60 & $3.51 \times 10^{-6}$ & 0.17 & $6.59 \times 10^{-7}$ & 9.41 \\
\hline$D R B 1 * 04$ & 1.00 & 1.21 & 0.01 & 0.35 & 0.18 & 3.50 \\
\hline$B * 08$ & 0.37 & 2.56 & 0.04 & 0.46 & 0.06 & 5. 61 \\
\hline$B * 58$ & 0.63 & 0.6 & 0.01 & 0.33 & 0.62 & 1.81 \\
\hline$D Q B 1 * 06$ & 1.00 & 1.86 & $4.14 \times 10^{-4}$ & 10.77 & 0.25 & 0.17 \\
\hline$D Q B 1 * 05$ & 0.00 & 0.19 & 0.14 & 1.66 & $4.51 \times 10^{-5}$ & 0.11 \\
\hline$D R B 1 * 16$ & 0.00 & 0.10 & 1.00 & 1.01 & 0.00 & 0.10 \\
\hline$B * 51$ & 0.45 & 0.47 & 0.22 & 1.67 & 0.07 & 0.27 \\
\hline
\end{tabular}

Hom = homozygous test (homozygous compared to absent); Het = heterozygous test (heterozygous compared to absent); Zyg = zygosity test (homozygous compared to heterozygous); $O R=$ odds ratio.

Zygosity test was performed using PyHLA programme. 
Table 3: Significant residue associations in the HLA-DRB1 pockets alleles in 73 diabetic seropositive children versus healthy controls

$\begin{array}{lccccc}\text { Position } & \text { Amino acid } & \text { Association } & \boldsymbol{P} \text { value } & \text { Corrected } \boldsymbol{P} & \text { Odds ratio } \\ \text { Pocket } 4[13,71,78,70,74,26] & & & & \\ 13 & \mathrm{~S} & + & 2.19 \times 10^{-13} & 1.69 \times 10^{-11} & 11.46 \\ 71 & \mathrm{~K} & + & 2.19 \times 10^{-13} & 1.69 \times 10^{-11} & 11.46 \\ 70 & \mathrm{~V} & + & 3.41 \times 10^{-13} & 2.63 \times 10^{-11} & 11.31 \\ \text { Pocket } 6[9,11,30] & & & & \\ 9 & \mathrm{E} & + & 1.98 \times 10^{-7} & 1.37 \times 10^{-5} & 5.43 \\ 11 & \mathrm{~S} & + & 1.04 \times 10^{-12} & 7.20 \times 10^{-11} & 10.43 \\ 30 & \mathrm{Y} & + & 6.92 \times 10^{-5} & 4.77 \times 10^{-3} & 12.29 \\ \text { Pocket } 7[28,61,71,47,67] & & & & 11.46 \\ 71 & \mathrm{~K} & + & 2.19 \times 10^{-13} & 1.51 \times 10^{-11} & \\ \text { Pocket 9 }[9,60,57,37,38] & & & & \\ 9 & \mathrm{E} & + & 1.98 \times 10^{-7} & 1.37 \times 10^{-5} & 5.43\end{array}$

Residue association test was performed using SKDM programme.

All the tested loci were in Hardy-Weinberg equilibrium in the cases considered for the present study, while they were not so in the controls. However, it must be noted that as this single-centre project was considered a preliminary study, tests to detect any potential associations were conducted.

Association test results indicated that the risk and protection of T1D in seropositive cases were associated with alleles belonging to HLA class I $(H L A-B)$ and class II (HLA-DRB1 and HLA-DQB1) genes [Table 1].

The strongest significant susceptibility alleles were found to be $H L A-D R B 1 * 03\left(P=9.19 \times 10^{-11}\right.$, OR = 4.97) and $D Q B 1 * 02\left(P=9.76 \times 10^{-8}, \mathrm{OR}=3.47\right)$. It was also observed that the seropositive cases for GADA (98.6\%), ICAs (23.3\%) and both the autoantibodies (21.9\%) have more $D R B 1 * 03$ or $D R B 1 * 04$ alleles
(95.8\%) than the seronegative cases (65.2\%) and healthy controls (39\%). However, the presence of risk alleles did not correlate with higher GADA autoantibody levels and the presence of protection alleles did not correlate with lower levels. Furthermore, seronegative cases showed significant risk association with $H L A$ $D R B 1 * 03$ and $-D Q B 1 * 02$ alleles, albeit to a lesser extent $\left(P=1.74 \times 10^{-3}, \mathrm{OR}=5.6\right.$ and $P=1.20 \times 10^{-2}$, $\mathrm{OR}=4.4)$.

The most significant resistance alleles were found to be $H L A-D Q B 1 * 06\left(P=6.40 \times 10^{-5}, \mathrm{OR}=0.05\right)$ and HLA-DQB1 *05 $\left(P=9.59 \times 10^{-5}, \mathrm{OR}=0.40\right)$.

The zygosity tests were performed to investigate homozygous, heterozygous and zygosity associations based on the genotype frequency differences in cases and controls. The results indicated that HLA-DRB1*03 and $D Q B 1{ }^{*} 02$ zygosity is associated with disease

Table 4: Correlations between lumbar spine bone mineral density t-score values and age, body mass index, haemoglobin levels and platelet-to-lymphocyte ratio, neutrophil-to-lymphocyte ratio and monocyte-to-lymphocyte ratio values

\begin{tabular}{|c|c|c|c|c|c|c|c|c|c|}
\hline \multicolumn{2}{|c|}{ Allele } & \multicolumn{4}{|c|}{$A$ independent of $B$} & \multicolumn{4}{|c|}{$B$ independent of $A$} \\
\hline Allele A & Allele B & $P 3$ & OR3 & $P 4$ & OR4 & P5 & OR5 & $P 6$ & OR6 \\
\hline \multicolumn{10}{|c|}{ Susceptibility } \\
\hline$B * 08$ & $D R B 1 * 03$ & 0.64 & 1.29 & 1 & 0.81 & $8.23 \times 10^{-4}$ & 15.67 & $1.95 \times 10^{-9}$ & 9.86 \\
\hline$D Q B 1 * 02$ & $D R B 1 * 03$ & 0.59 & 2.22 & 0.25 & 1.91 & $3.43 \times 10^{-6}$ & 7.83 & 0.10 & 6.76 \\
\hline \multicolumn{10}{|l|}{ Resistance } \\
\hline$D Q B 1 * 05$ & $D R B 1 * 16$ & 0.61 & 0.52 & 0.02 & 0.33 & 0.50 & 1.45 & 1 & 0.92 \\
\hline
\end{tabular}

$L D=$ linkage disequilibrium; $O R=$ odds ratio.

Interaction tests were performed using PyHLA programme.

If both P3 and P4 are significant, then A is associated with type 1 diabetes (T1D) independently of B; If P5 and P6 are significant, then B is associated with T1D independently of $A$; if both P3 and P5 are significant, then $A$ and B show interaction in T1D; if P7 is significant, then the difference between A and B is associated with T1D; if P8 is significant, then A and B have combined action; if P9 is significant, then A and B are in linkage disequilibrium (LD) in cases; if $P 10$ is significant, then $A$ and $B$ are in $L D$ in controls. 
Table 4 (cont'd): Correlations between lumbar spine bone mineral density t-score values and age, body mass index, haemoglobin levels and platelet-to-lymphocyte ratio, neutrophil-to-lymphocyte ratio and monocyte-to-lymphocyte ratio values

\begin{tabular}{|c|c|c|c|c|c|c|c|c|c|}
\hline \multicolumn{2}{|c|}{ Allele } & \multicolumn{2}{|c|}{ Difference } & \multicolumn{2}{|c|}{ Combined action } & \multicolumn{2}{|c|}{ LD in cases } & \multicolumn{2}{|c|}{ LD in controls } \\
\hline Allele A & Allele B & $P 7$ & OR7 & $P 8$ & OR8 & $P 9$ & OR9 & $P 10$ & OR10 \\
\hline \multicolumn{10}{|c|}{ Susceptibility } \\
\hline$B * 08$ & $D R B 1 * 03$ & 0.00 & 0.08 & $1.57 \times 10^{-8}$ & 12.71 & 0.02 & 6.4 & 0.01 & 4.03 \\
\hline$D Q B 1 * 02$ & $D R B 1 * 03$ & 0.24 & 0.28 & $1.66 \times 10^{-12}$ & 14.99 & $1.76 \times 10^{-5}$ & 25.61 & $1.32 \times 10^{-8}$ & 22.13 \\
\hline \multicolumn{10}{|l|}{ Resistance } \\
\hline$D Q B 1 * 05$ & $D R B 1 * 16$ & 0.56 & 0.36 & 0.03 & 0.48 & $1.76 \times 10^{-10}$ & 47.24 & $8.83 \times 10^{-11}$ & 29.94 \\
\hline
\end{tabular}

$L D=$ linkage disequilibrium; $O R=$ odds ratio.

Interaction tests were performed using PyHLA programme.

If both P3 and P4 are significant, then A is associated with type 1 diabetes (T1D) independently of B; If P5 and P6 are significant, then B is associated with T1D independently of A; if both P3 and P5 are significant, then A and B show interaction in T1D; if P7 is significant, then the difference between A and B is associated with T1D; if P8 is significant, then A and B have combined action; if P9 is significant, then A and B are in linkage disequilibrium (LD) in cases; if $P 10$ is significant, then $A$ and $B$ are in $L D$ in controls.

susceptibility $(P<0.0001$, OR $=8.22$ and $P<0.0001$, OR $=9.41$, respectively). In other words, compared to controls, the cases had a significantly higher frequency of risk allele homozygous genotypes than risk allele absent genotypes [Table 2].

Notably, heterozygous genotypes DRB1*03/04 and $D Q B 1 * 02 / 03$ were associated with significant T1D risk $(P<0.0001, \mathrm{OR}=63.21$ and $P=0.02$, OR $=$ 3.63 , respectively). However, heterozygosity, i.e. the higher frequency of risk allele $(B * 08, B * 58, D R B 1 * 03$, $D Q B 1 * 02$ and $D R B 1 * 04$ ) heterozygous genotypes than risk allele absent genotypes in cases, was associated with disease protection $(P=0.04, \mathrm{OR}=0.46 ; P=0.01$, $\mathrm{OR}=0.33, P<0.0001, \mathrm{OR}=0.07 ; P<0.0001, \mathrm{OR}=0.17$; and $P=0.01, \mathrm{OR}=0.35$, respectively).

T1D protection was associated with the zygosity of protective alleles $D R B 1^{*} 16(P=0.0013$, OR $=$

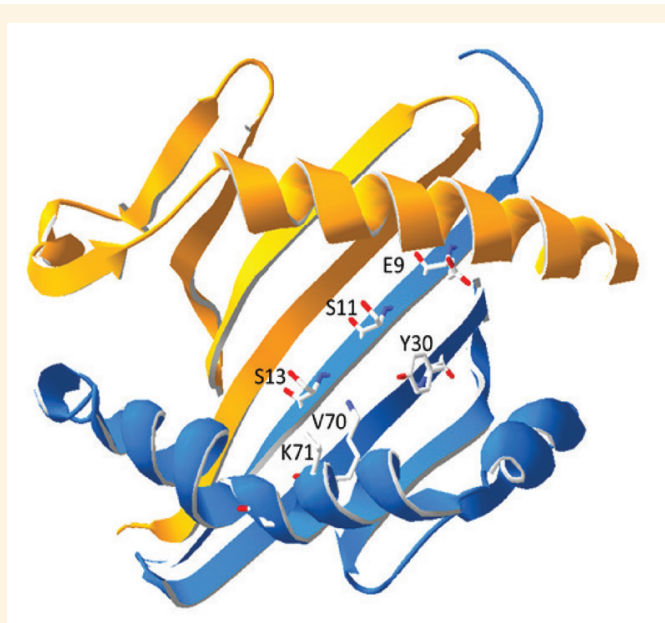

Figure 1: Ribbon model of the peptide-binding groove of a Human Leukocyte Antigen - DR isotype molecule, showing the position and the side-chain of significantly associated residues. The model was based on 3pdo entry from Protein Data Bank and the figure was prepared using Swiss-PdbViewer. ${ }^{31,32}$
$0.10)$ and $D Q B 1 * 05\left(P=4.51 \times 10^{-5}, \mathrm{OR}=0.11\right)$ and T1D susceptibility was associated with $D Q B 1 * 06$ heterozygosity $\left(P=4.14 \times 10^{-4}\right.$, OR $\left.=10.77\right)$.

Since HLA genotypes dictate the affinity to the presented peptides, the T1D-associated HLA alleles are implicated in the selective presentation of selfpeptides; therefore, the residues in the HLA chains with potential associations were investigated using the pocket test. The results showed that six residues

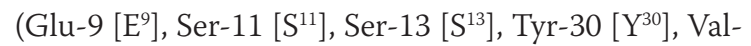
$70\left[\mathrm{~V}^{70}\right]$ and Lys-71 $\left.\left[\mathrm{K}^{71}\right]\right)$ in pockets $4,6,7$ and 9 of the HLA class II DRB1 chain were significantly associated with T1D susceptibility [Table 3 and Figure 1].

The zygosity analysis for five associated residues $\left(E^{9}, S^{11} S^{13}, V^{70}, K^{71}\right)$ showed that only the heterozygotes were associated with T1D susceptibility $(P=1.547 \times$ $10^{-7}, \mathrm{OR}=6.04 ; P=3.13 \times 10^{-12}, \mathrm{OR}=10.43 ; P=3.13$ $\times 10^{-12}, \mathrm{OR}=10.43 ; P=7.357 \times 10^{-13}, \mathrm{OR}=11.68$ and $P=3.13 \times 10^{-12}, \mathrm{OR}=10.43$, respectively). In contrast, residue $\mathrm{Y}^{30}$ homozygotes $\left(P=1.199 \times 10^{-7}\right.$, OR $=$ 33.65), heterozygotes $(P=0.02305, \mathrm{OR}=6.7)$ and

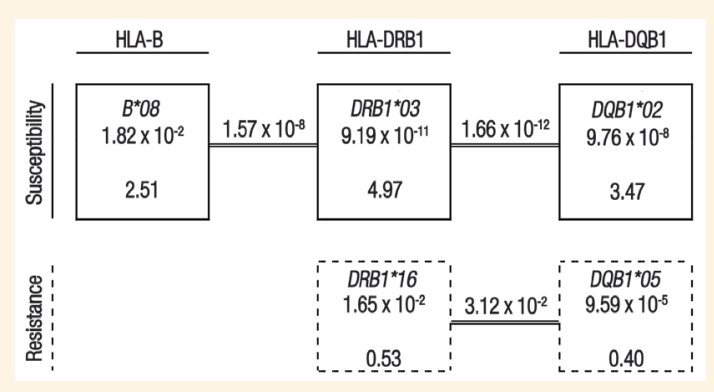

Figure 2: A representation of detected combined actions between T1D susceptibility and resistance alleles of the human leukocyte antigen genes with corrected $P$ values in the top-half of the boxes and odds ratios on the bottom-half. The lines connecting the gene alleles represent combined actions, with $P$ values above each of these lines. 
zygosity $\left(P=8.753 \times 10^{-6}, \mathrm{OR}=5.02\right)$ were all found to be associated with T1D susceptibility.

Since T1D association with HLA alleles were reported in the haplotypic context as well as the genotypic context, the associated allele interactions were also analysed in the present study. Two haplotypes were found to be associated with risk (HLA-B*08-DRB1*03: P8 = $1.57 \times 10^{-8}, \mathrm{OR}=12.71$ and HLA-DRB1*03-DQ*02: P8 = $1.66 \times 10^{-12}$, OR = 14.99) [Table 4 and Figure 2]. However, the interaction analysis indicated that $D R B 1 * 03$ association with T1D was independent of $B^{*} 08\left(P 5=8.23 \times 10^{-4}, P 6=\right.$ $\left.1.95 \times 10^{-9}\right)$, while $B^{*} 08$ association is dependent $(P 3$ $=0.64, P 4=1)$. Additionally, both alleles were found to have a combined effect in the disease $(P 8=1.57 \times$ $10^{-8}$ ) [Table 4]. Furthermore, the data indicated that a combined-dependent effect of the HLA-DRB1*03$D Q * 02$ haplotype resulted in T1D susceptibility, while a combined-dependent effect of the DRB1*16$D Q B 1 * 05$ haplotype resulted in protection [Table 4 and Figure 2].

\section{Discussion}

The risk and protection of T1D among the Omani population are associated with alleles belonging to the $H L A-B, H L A-D R B 1$ and $H L A-D Q B 1$ genes, an observation that is consistent with those reported in other populations [Table 1]. ${ }^{8}$ This was expected as the Omani population is genetically related to the Arab, Mediterranean and West-Asian populations. . 12,13,17 $^{-17}$

The HLA class I alleles associated with T1D susceptibility were $B^{*} 08\left(P=1.82 \times 10^{-2}\right.$, OR $=$ $2.51)$ and $B^{*} 58\left(P=2.86 \times 10^{-2}, \mathrm{OR}=2.47\right)$ and that associated with protection was $B^{*} 51\left(P=1.82 \times 10^{-2}\right.$, $\mathrm{OR}=0.41)$. These findings are consistent with those reported in previous studies. ${ }^{18}$ The association of $B * 08$ with autoimmune diseases was attributed to its presence in LD with $D R B 1 * 03$, which was observed in both cases and controls [Table 4] ${ }^{18}$ Furthermore, results indicated that $B * 08$ association is dependent on $D R B 1 * 03$. Additionally, $B * 58$ is part of a significantly associated haplotype among the North Indian and Han Chinese populations and results from both these populations suggest that the association is not attributed to the allele itself. ${ }^{19,20}$

As predicted by a previous study, T1D protection in Omanis was found to be associated with HLADR2 (DRB1*16) and DQ1 (DQB1*05 and DQB1*06) alleles. ${ }^{14}$ The highest significant resistance alleles are HLA-DQB1*06 $\left(P=6.40 \times 10^{-5}\right.$, OR $\left.=0.05\right)$ and HLA-DQB1*05 $\left(P=9.59 \times 10^{-5}, \mathrm{OR}=0.40\right)$. However, despite the high frequency of the $D R B 1 * 16$ allele in the Omani population compared to other populations, its significant association with protection was relatively weaker $(P=0.02$, OR $=0.5) .{ }^{21}$ This was likely due to the presence of different alleles (DRB1*16:01:01, 16:02:01 and $16 * 64$; personal communication) in the Omani population that are not all protective.

Notably, about $96 \%$ of the seropositive cases were observed to have either the $D R B 1 * 03$ or $D R B 1 * 04$ allele, but the presence of these alleles were not associated with higher GADA autoantibody levels. Furthermore, no association was detected between GADA autoantibody levels and risk or protection genotypes.

The zygosity test showed that the HLA-DRB1*03 and $D Q B 1 * 02$ zygosity were associated with risk $(P<0.0001$, OR $=0.08)$ while heterozygosity was associated with protection $(P<0.0001$, OR $=0.17$, indicating that the risk associated with both alleles is recessive, as suggested by others. ${ }^{7}$ Furthermore, it was also detected that the heterozygous genotypes, DRB1 *03/04 $(P<0.0001, \mathrm{OR}=63.21)$ and $D Q B 1 * 02 / 03$ $(P=0.02, \mathrm{OR}=3.63)$, were associated with significant T1D risk.

In contrast, the protection associated with heterozygosity of the same risk-associated alleles may be attributed to the presence of protection alleles in the genotypes. A total of 27 of the 44 heterozygous $H L A$ $D R B 1 * 03$ cases had one of the HLA-DR2 protectionassociated alleles (five cases with $D R B 1 * 15$ and 22 with $D R B 1 * 16)$ and 30 of the heterozygous HLA-DQB1*02 cases ( $\mathrm{n}=39$ ) had one of the HLA-DQ1 protectionassociated alleles (29 cases with DQB1*05 and one with $D Q B 1 * 06)$.

Additionally, the zygosity test showed that the protection associated with $D Q B 1 * 05$ and $D R B 1 * 16$ were significant in homozygosity, suggesting that the protection associated with both the alleles was recessive.

The side chains of self-peptide residues' interaction with the binding groove pockets stabilise the peptide-HLA class II complex and are therefore known as anchor residues. The binding grooves of HLA class II chains are characterised by the properties of the P1, P4, P6 and P9 pockets that lend specificity to the anchor residues. ${ }^{22} \mathrm{~T} 1 \mathrm{D}$-associated residues 9, 11,13 and 30 are located in the $B$-sheet floor, with their side chains in the peptide-binding groove, while residues 70 and 71 are in the $\alpha$-helix, with their side chains close to residue 13 [Figure 1]. DRB1 $\mathrm{S}^{13}$ is in pocket $4, \mathrm{~K}^{71}$ in pockets 4 and $7, \mathrm{~V}^{70}$ in pocket $4, \mathrm{~S}^{11}$ in pocket $6, \mathrm{E}^{9}$ in pockets 6 and 9 and $\mathrm{Y}^{30}$ in pocket 6 . As $S^{13}, V^{70}$ and $K^{71}$ were found to be associated with the strongest disease risk based on the $P$ values and OR values, they might be the major contributors from pocket 4 . 
The association of $\mathrm{S}^{13}$ and $\mathrm{K}^{71}$ with T1D susceptibility was reported by previous studies and they were implicated in joint susceptibility to both T1D and autoimmune thyroid disease. ${ }^{23-25}$ The $S^{11}$, $\mathrm{S}^{13}$ and $\mathrm{K}^{71}$ residues were also associated with risk of rheumatoid arthritis. ${ }^{26}$ This suggests common disease mechanisms that operate irrespective of the presented self-peptides.

Transgenic mice expressing T1D human class II susceptibility alleles showed that MHC class II molecules present specific autoantigenic peptides, such as GAD65 peptides, which can potentially activate autoreactive CD4+ $\mathrm{T}$ cells that are known to assist in targeting $B$ cells by cytotoxic CD8+ and autoantibody-producing B cells. ${ }^{27}$

Interaction tests suggested that the association of HLA-DRB1*03 and -DQB1*02 haplotype with T1D risk resulted from a combined-dependent effect [Table 4]. Notably, $78 \%$ of cases with this haplotype were GADA positive, as reported by previous studies. ${ }^{28}$ This indicated that both susceptibility HLA alleles and antiGAD were risk factors for T1D. However, an association between risk alleles and higher GADA levels was not detected. This may indicate that GADA autoantibody level, which is implicated in the destructive process in the islets, is not genetically driven.

Furthermore, the analysis indicated that the association with T1D resulted from a combineddependent effect of the DRB1*16-DQB1*05 haplotype [Table 4]. This haplotype is thought to have a protective role, but due to its rare occurrence in Caucasians and East Asians, its effect in T1D resistance could not be proven. Additionally, the present study also posited that $D R B 1 * 16-D Q B 1 * 05$ haplotype in the Omani population could potentially protect autoantibody seropositive first-degree relatives from T1D, similar to the HLA-DRB1*15:01-DQB1*06:02 haplotype in other populations. ${ }^{6}$

Although other T1D-associated haplotypes such as $D R B 1 * 04-D Q B 1 * 03$ (7.7\%), DRB1*07-DQB1*02 (6.4\%) and $D R B 1 * 15-D Q B 1 * 06$ (1\%) were reported in the Omani population, the present study did not detect significant LD in the investigated group of cases and controls, which is likely due to the small sample size. $^{12}$

Notably, the frequency of seronegative cases (26\%) was found to be higher than what was reported in other ethnic groups (20\%). ${ }^{29}$ However, a relatively weaker association of T1D with $H L A-D R B 1$ and $-D Q B 1$ alleles in seronegative cases may reflect the fact that some of the cases may be positive for other autoantibodies associated with T1D that were not tested for in this study or that a positive result might be obtained on repeat testing, as reported by Hameed et al..$^{30}$
A major limitation of the present study was its small sample size as it was based on data from a single centre. Therefore, it is recommended that, in order to verify the results of the preliminary study, a larger, multi-centre study be conducted by at least doubling the sample size of the cases and increasing the controlsto-cases ratio (at least 3:1) to reach acceptable power ( $\geq 80 \%$ ). In addition, sequencing of the associated risk and protection allele should be considered.

\section{Conclusion}

The majority of the seropositive T1D cases (71\%) have a family history of T1D and/or T2D. Despite its small sample size, the present study identified $D Q B 1 * 02, D R B 1 * 03$ and DRB1*04 as potential risk alleles in GADA and/or ICA seropositive T1D in Omani children. In addition, the study observed an association of the DRB1*16-DQB1*05 haplotype with $\mathrm{T} 1 \mathrm{D}$ protection in a combined-dependent manner.

\section{AUTHORS' CONTRIBUTION}

MA-B, AA-J, SH and ES developed the proposal. MAB, SA-B, AA-S, SA-H and AA collected the data. MA-B and HA-R ordered the required materials. MA-B and SA-B conducted the laboratory work. SA-Y reviewed the clinical and family histories. SA-B and AA-A analysed the data. AA-A drafted the manuscript. MA-B and SA-Y revised the manuscript. All authors approved the final version of the manuscript.

\section{ACKNOWLEDGEMENTS}

We would like to thank all the patients and their parents. We also acknowledge the support offered by Dr Irfan Ullah from the Pediatric Department at SQUH and Ms Faiza Al-Yahyai, Ms Faiza Al-Ghanami and Ms Iman Al-Hadhili from the genetic lab. We express special gratitude to Dr Felix Fan and Dr Abdelhamid Abdesselam for their assistance with the utilisation of pyHLA.

\section{CONFLICT OF INTEREST}

The authors declare no conflict of interest.

\section{FUNDING}

This work was supported by The Research Council fund, Oman (RClMEDIMICR114101).

\section{References}

1. Mobasseri M, Shirmohammadi M, Amiri T, Vahed N, Fard HH. Prevalence and incidence of type 1 diabetes in the world: A systematic review and meta-analysis. Health Promot Perspect 2020; 10:98-115. https://doi.org/10.34172/hpp.2020.18. 
2. Stankov K, Benc D, Draskovic D. Genetic and epigenetic factors in etiology of diabetes mellitus type 1. Pediatrics 2013; 132:1112-22. https://doi.org/10.1542/peds.2013-1652.

3. Wållberg M, Cooke A. Immune mechanisms in type 1 diabetes. Trends Immunol 2013; 34:583-91. https://doi.org/10.1016/j. it.2013.08.005

4. Jacobsen LM, Newby BN, Perry DJ, Posgai AL, Haller MJ, Brusko TM. Immune mechanisms and pathways targeted in type 1 diabetes. Curr Diab Rep 2018; 18:90. https://doi. org/10.1007/s11892-018-1066-5.

5. Lam H V., Nguyen DT, Nguyen CD. Sibling method increases risk assessment estimates for type 1 diabetes. PLoS One 2017; 12:e176341. https://doi.org/10.1371/journal.pone.0176341.

6. Pugliese A. Autoreactive T cells in type 1 diabetes. J Clin Invest 2017; 127:2881-91. https://doi.org/10.1172/JCI94549.

7. Dib SA, Gomes MB. Etiopathogenesis of type 1 diabetes mellitus: Prognostic factors for the evolution of residual $\beta$ cell function. Diabetol Metab Syndr 2009; 1:25. https://doi. org/10.1186/1758-5996-1-25

8. Noble JA. Immunogenetics of type 1 diabetes: A comprehensive review. J Autoimmun 2015; 64:101-12. https://doi. org/10.1016/j.jaut.2015.07.014.

9. Zayed H. Genetic epidemiology of type 1 diabetes in the 22 Arab countries. Curr Diab Rep 2016; 16:37. https://doi. org/10.1007/s11892-016-0736-4.

10. Soliman AT, Al-Salmi IS, Asfour MG. Epidemiology of childhood insulin-dependent diabetes mellitus in the Sultanate of Oman. Diabet Med 1996; 13:582-6. https://doi.org/10.1002/ (SICI)1096-9136(199606)13:6<582::AID-DIA114>3.0.CO;2-E.

11. Al-Yaarubi S, Ullah I, Sharef SW, Al Shidhani A, Al Hanai S, Al Kalbani R, et al. Demographic and clinical characteristics of type 1 diabetes mellitus in Omani children - single center experience. Oman Med J 2014; 29:119-22. https://doi. org/10.5001/omj.2014.29.

12. Albalushi KR, Sellami MH, AlRiyami H, Varghese M, Boukef MK, Hmida S. HLA class II (DRB1 and DQB1) polymorphism in Omanis. J Transplant Technol Res 2014; 4. https://doi. org/10.4172/2161-0991.1000134.

13. Al Salmi I, Metry A, Al Ismaili F, Hola A, Shaheen F, Fakhoury $\mathrm{H}$, et al. Epidemiology of human leukocyte antigens among Omani population. Saudi J Kidney Dis Transplant 2017; 28:1021-26. https://doi.org/10.4103/1319-2442.215135.

14. White AG, Leheny W, Kuchipudi P, Varghese M, Al Riyami $\mathrm{H}, \mathrm{Al}$ Hashmi S, et al. Histocompatibility antigens in Omanis: Comparison with other Gulf populations and implications for disease association. Ann Saudi Med 1999; 19:193-6. https:// doi.org/10.5144/0256-4947.1999.193.

15. Fan Y, Song YQ. PyHLA: Tests for the association between HLA alleles and diseases. BMC Bioinformatics 2017; 18:90. https://doi.org/10.1186/s12859-017-1496-0.

16. Kanterakis S, Magira E, Rosenman KD, Rossman M, Talsania K, Monos DS. SKDM human leukocyte antigen (HLA) tool: A comprehensive HLA and disease associations analysis software. Hum Immunol 69, 522-5. https://doi.org/10.1016/j. humimm.2008.05.011.

17. Jahromi M, Al-Ozairi E. Human leukocyte antigen (HLA) and islet autoantibodies are tools to characterize type 1 diabetes in Arab countries: Emphasis on Kuwait. Dis Markers 2019; 2019:9786078. https://doi.org/10.1155/2019/9786078.

18. Sia C, Weinem $M$. The role of HLA class i gene variation in autoimmune diabetes. Rev Diabet Stud 2005; 2:97-109. https:// doi.org/10.1900/rds.2005.2.97.
19. Zhang J, Zhao L, Wang B, Gao J, Wang L, Li L, et al. HLA$A * 33-D R 3$ and $A * 33-D R 9$ haplotypes enhance the risk of type 1 diabetes in Han Chinese. J Diabetes Investig 2016; 7:514-21. https://doi.org/10.1111/jdi.12462.

20. Kumar N, Mehra NK, Kanga U, Kaur G, Tandon N, Chuzho $\mathrm{N}$, et al. Diverse human leukocyte antigen association of type 1 diabetes in north India. J Diabetes 2019; 11:719-28. https://doi. org/10.1111/1753-0407.12898.

21. Gomes KFB, Santos AS, Semzezem C, Correia MR, Brito LA, Ruiz MO, et al. The influence of population stratification on genetic markers associated with type 1 diabetes. Sci Rep 2017; 7:43513. https://doi.org/10.1038/srep43513.

22. Jones EY, Fugger L, Strominger JL, Siebold C. MHC class II proteins and disease: A structural perspective. Nat Rev Immunol 2006; 6:271-82. https://doi.org/10.1038/nri1805.

23. Hu X, Deutsch AJ, Lenz TL, Onengut-Gumuscu S, Han B, Chen WM, et al. Additive and interaction effects at three amino acid positions in HLA-DQ and HLA-DR molecules drive type 1 diabetes risk. Nat Genet 2015; 47:898-905. https://doi. org/10.1038/ng.3353.

24. Redondo MJ, Steck AK, Pugliese A. Genetics of type 1 diabetes. Pediatr Diabetes 2018; 19:346-53. https://doi.org/10.1111/ pedi.12597.

25. Menconi F, Monti MC, Greenberg DA, Oashi T, Osman R, Davies TF, et al. Molecular amino acid signatures in the MHC class II peptide-binding pocket predispose to autoimmune thyroiditis in humans and in mice. Proc Natl Acad Sci 2008; 105:14034-9. https://doi.org/10.1007/978-1-62703-197-4-9.

26. Raychaudhuri S, Sandor C, Stahl EA, Freudenberg J, Lee HS, Jia $\mathrm{X}$, et al. Five amino acids in three HLA proteins explain most of the association between $\mathrm{MHC}$ and seropositive rheumatoid arthritis. Nat Genet 2012; 44:291-6. https://doi.org/10.1038/ ng.1076.

27. James EA, Mallone R, Kent SC, Dilorenzo TP. T-cell epitopes and neo-epitopes in type 1 diabetes: A comprehensive update and reappraisal. Diabetes 2020; 69:1311-35. https://doi. org/10.2337/dbi19-0022.

28. Krischer JP, Liu X, Lernmark Å, Hagopian WA, Rewers MJ, She JX, et al. The influence of type 1 diabetes genetic susceptibility regions, age, sex, and family history on the progression from multiple autoantibodies to type 1 diabetes: A TEDDY study report. Diabetes 2017; 66:3122-9. https://doi.org/10.2337/ db17-0261.

29. Wang J, Miao D, Babu S, Yu J, Barker J, Klingensmith G, et al. Prevalence of autoantibody-negative diabetes is not rare at all ages and increases with older age and obesity. J Clin Endocrinol Metab 2007; 92:88-92. https://doi.org/10.1210/jc.2006-1494.

30. Hameed S, Ellard S, Woodhead HJ, Neville KA, Walker JL, Craig ME, et al. Persistently autoantibody negative (PAN) type 1 diabetes mellitus in children. Pediatr Diabetes 2011; 12:142-9. https://doi.org/10.1111/j.1399-5448.2010.00681.x.

31. Protein Data Bank. Research Collaboratory for Structural Bioinformatics. From: https://www.rcsb.org Accessed: Dec 2021

32. Guex N, Diemand A, Peitsch MC, Schwede T. SwissPdbViewer. From: https://spdbv.unil.ch Accessed: Dec 2021 